\title{
Nano superconductivity and quantum processing of information in living organisms
}

\author{
Pavlo Mikheenko \\ Department of Physics \\ University of Oslo \\ Oslo, Norway \\ pavlo.mikheenko@fys.uio.no
}

\begin{abstract}
With the advance of superconducting quantum computing and the attempts of extending its operating range to higher temperatures, a special attention is paid to nanostructured quantum circuits. In particular, quasi onedimensional quantum wires with phase slip centers are argued to be promising structures for the next generation of quantum computers. In its turn, this stimulates revisiting the question about the possibility of quantum processing of information in quasi one-dimensional structures in the nervous system, specifically the brain, in living organisms, especially in the light of recent findings that suggest robust room-temperature superconductivity in these structures. The early theories of superconductivity were in favor of its quasi one-dimensional nature, and the recent findings suggest that reducing dimensions of a system could be a good approach for increasing the critical temperature of a material. Here, based on experimental data, it is argued that both room-temperature superconductivity and quantum processing of information are possible in the microtubules that are abundant in the nervous system and form the scaffolding of every cell in advanced living organisms. The origin of superconductivity in microtubules, and the way in which quantum processing of information may take place in them, are discussed. The role of Josephson oscillations in processing and exchange of information is emphasized.
\end{abstract}

Keywords-Superconductivity, quantum processing of information, nanostructures, microtubules, Josephson oscillations

\section{INTRODUCTION}

Since the discovery of superconductivity in 1911 , there were many attempts to explain its nature. A good account of these attempts is presented in [1]. Most of the prominent scientists favored one-dimensional theories. For example, Albert Einstein in 1922 was suggesting molecular conduction chains, in which electrons undergo continuous cyclic exchanges. Kamerlingh Onnes, discoverer of superconductivity, was hypothesizing about superconducting filaments, and Joseph John Thompson, discoverer of the electron, was considering fluctuating electric dipole chains as an explanation of superconductivity. In [1], these were named 'failed theories of superconductivity', admitting their interesting and inspiring aspects. It is possible, however, that intuition of the scientists was correct and the superconductivity at its best, namely with highest critical temperature, is in its one-dimensional, or, more correctly, quasi one-dimensional form. A remarkable theory of quasi one-dimensional superconductivity was developed by
William Little in 1964, suggesting electron-electron interaction as its basis and deriving very high effective critical temperature of $\approx 2200 \mathrm{~K}$ in linear chains of organic molecules interacting with connected to them molecular complexes [2]. While it is understandable that molecular chains will not survive at such a high temperature, it does not mean that superconductivity with this mechanism cannot exist at room temperature, at which molecular chains are stable. Moreover, due to large difference between critical and room temperature, one could expect microscopic quantum tunneling in the junctions made of such superconductor and, based on this, quantum processing of information with them. A hypothesis was put forward in [3] that such superconductivity was 'found' by chance by nature billions of years ago and was developed through evolution, resulting in the intelligent life forms we have on the planet now. The only purpose of developing superconductivity was quantum processing of information, which was giving advantages to specific organisms in survival and reproduction.

The idea of room-temperature superconductivity in living organisms, specifically in the nervous system and the brain, as part of it, was given by Halperin and Wolf in 1973 [4]. In [3], experimental evidences in favor of room-temperature superconductivity were given, estimating the critical temperature, which appeared to be close to that derived theoretically by William Little [2]. The superconductivity was assigned to microtubules abundant in nervous system and present in cells of living organisms. The existence of superconductivity in microtubules would allow for quantum processing of information in them at room temperature following, for example, ideas suggested earlier by Penrose and Hameroff [5].

This paper deals with the analyses of experimental results obtained by electrical transport measurements of the slices of rat and pig brains. The results are discussed in light of recently published article [6], which considers nanostructured quantum circuits based on quasi onedimensional quantum wires with phase slip centers. These quantum circuits appear promising in extending the operating range of quantum computers to higher temperatures. It is argued that such circuits, based on microtubules, are already in functioning in living organisms. It is suggested that, as in quantum computers, they use Josephson effect, contributing nonlinearity to inductancecapacitance oscillations in the quantum circuits [7]. The role of Josephson oscillations in the processing and exchange of information in living organisms will be discussed. 


\section{EXPERIMENTAL}

\section{A. Electrical transport measurements of brain slices}

Electrical transport measurements were done by a fourelectrode, and in some cases three-electrode technique using electrodes set on an insulating substrate, to which brain slices were tightly pressed after being wrapped in a polymer film to keep water balance in the samples [3]. The main idea of experiments was to use a quantum mediator to allow the transfer of information from a quantum object to a classical measurement system. This mediator was graphene [8]. It satisfies all requirements for the measurements, being highly conductive, compatible with the wet environment of the samples, and behaving quantum-mechanically at room temperature. Before the measurements, brain slices were soaked in aqueous solution of graphene nano-flakes. The commercial solution was obtained from Sigma-Aldrich, with concentration of the graphene nano-flakes of $1 \mathrm{mg}$ per $\mathrm{mL}$ of water. Different brain slices were exposed to the graphene solution for different durations of time, from one minute to three hours, some being also subjected to ultrasound vibrations to accelerate penetration of graphene nano-flakes into the slices. The rat brain slices were of the thickness of $40 \mu \mathrm{m}$, while slices of pig brains were of 1-mm thickness.

The electrodes were wires approximately $1 \mathrm{~cm}$ in length and of the thickness of $0.2-0.5 \mathrm{~mm}$, made of different materials: gold, copper and copper covered with a $\mathrm{Pb}-\mathrm{Sn}$ alloy. All of them provided similar results. With these electrodes, millions of microtubules were connected to them in parallel via graphene nano-flakes, providing statistically averaged data. The focus of the research was on measuring current-voltage characteristics of the slices.

A range of control experiments was carried out to ensure that the observed effects are not of instrumental nature or not coming from the graphene itself. These experiments are described in [3].

\section{RESULTS AND DISCUSSION}

\section{A. Evidence of phase slip lines in the brain slices}

Fig. 1 shows the current-voltage characteristic of a narrow (approximately $50 \mu \mathrm{m}$ in width) segment of rat brain slice.

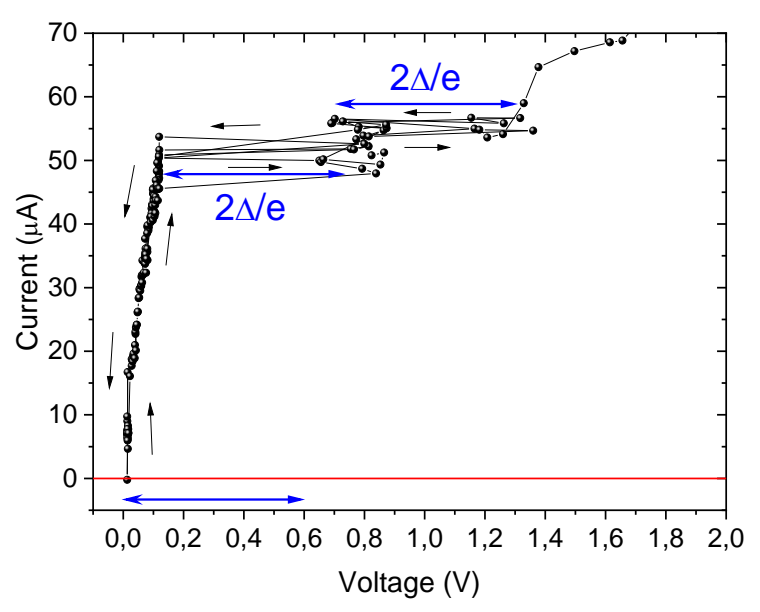

Fig. 1. Current-voltage characteristic of a narrow segment of rat brain slice. The observed voltage jumps of the length of double energy gap of superconductor divided to the charge of electron reflect appearance of phaseslip lines.
In the characteristic, there is a dissipation-free part with zero voltage lasting up to about $15 \mu \mathrm{A}$, a resistive part extending from 15 to $53 \mu \mathrm{A}$ and, most importantly, jumps in the voltage of the size of about $0.6 \mathrm{~V}$, which are reproducible in length at the increase and decrease of current. These jumps were recorded several times. Their approximate length is shown in the plot by the blue-line fragments with arrows at both sides. One of the fragments is placed close to the voltage axis. The red line shows position of the zero current. In one and two-dimensional systems, such jumps correspond to phase-slip centers and phase-slip lines, respectively [6,9]. In [10], it was found that the minimal value of these jumps is $2 \Delta / \mathrm{e}$, which is equal to the voltage of two nearly merged superconductor - normal metal boundaries. Here $\Delta$ is the energy gap of the superconductor and $\mathrm{e}$ is the charge of the electron. Since there are no larger jumps of voltage in the samples, and a considerable hysteresis of current-voltage characteristics is absent too, one can suggest that these jumps correspond to phase-slip lines. The length of the jumps indeed corresponds to $2 \Delta / \mathrm{e}$, as was found in [3].

In Fig. 2, the current-voltage characteristic of a wide (approximately $2.5 \mathrm{~mm}$ in width) section of rat brain slice is shown. In spite of a nearly three orders of magnitude difference in current (tens of microvolts in Fig. 1 and tens of

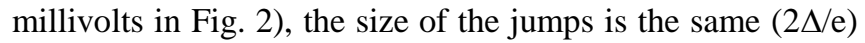
in both plots, as marked by identical blue arrowed fragments. This current-voltage characteristic was recorded in the threeelectrode configuration. The initial slope of the curve corresponds to the contact resistance between sample and the common current-voltage electrode. The contribution of the contact resistance to the current-voltage characteristic is not large.

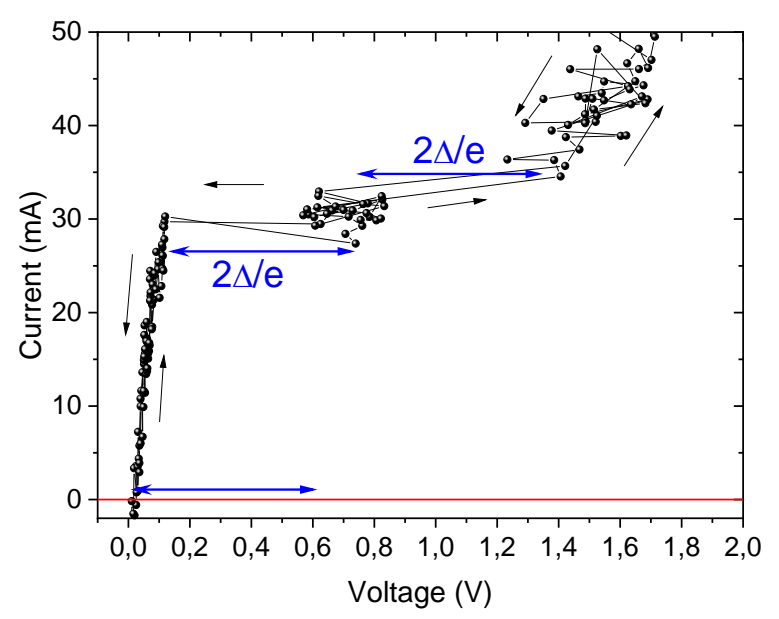

Fig. 1. Current-voltage characteristic of a wide section of rat's brain slice. The observed voltage jumps reflect appearance of phase-slip lines.

Moreover, a slice of pig brain shows the same features as in Figs. 1 and 2, as displayed in Fig. 3. The current-voltage characteristic in this plot is recorded using the four-electrode technique. A small slope of its initial part is due to the overlap of dissipation-free branch with finite conductivity of graphene nano-flakes penetrating cell membranes and connecting microtubules. The image in Fig. 3 reflects appearance of at least two phase-slip lines. Their formation is 
quite specific. About 1000 experimental points were recorded in this experiment, slowly increasing and decreasing the current in the dissipation-free part, before formation of phase-slip lines took place. There were three attempts of their formation at the current marked in the plot by the higher horizontal red arrow, and possibly one attempt shown by the lower horizontal red arrow.

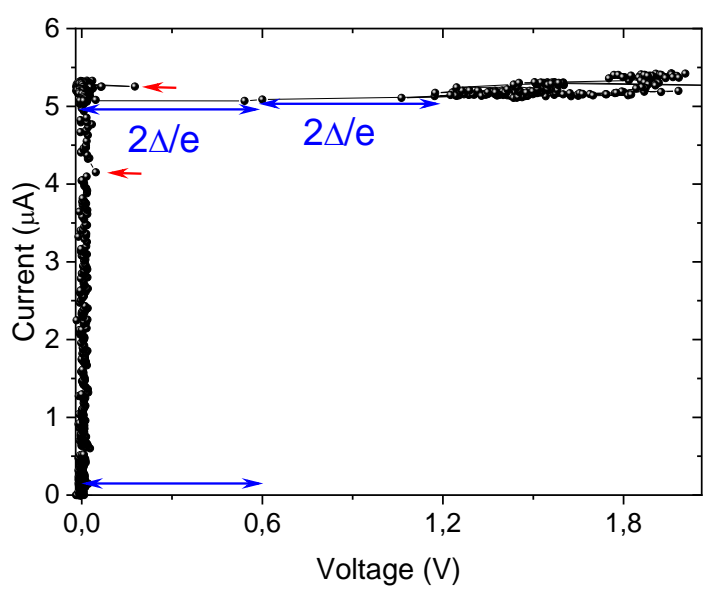

Fig. 3. Current-voltage characteristic of a slice of pig's brain. The observed voltage jumps of the length of double energy gap of superconductor divided to the charge of electron reflect appearance of phase-slip lines. Horizontal red arrows mark the attempts of the formation of the phase-slip lines.

These attempts are consistent with quantum behavior of phase-slip lines in a nanowire made of high-temperature superconductor $\mathrm{YBa}_{2} \mathrm{Cu}_{3} \mathrm{O}_{7-\mathrm{x}}$ described in [6]. It shows a certain probability of the switch from superconducting to resistive state from the different quantized energy levels of the system. The fact that formation of phase-slip lines happened not at the maximum current means that the switch of the system took place not from the ground energy level, but from an excited level [6].

One needs to remember that the description of Figs. 1-3 deals with superconductivity at room temperature in biological systems, namely brain slices, most of which were fixed in formalin and all of which were soaked in the aqueous solution of graphene nano-flakes [3]. In [6], it was emphasized that phase-slip lines with quantized energy levels are ideal objects for quantum processing of information taking place at an elevated temperature, which is much higher than in currently developed quantum computers. In particular, for the high-temperature superconductor $\mathrm{YBa}_{2} \mathrm{Cu}_{3} \mathrm{O}_{7-\mathrm{x}}$ with suggested critical temperature of $90 \mathrm{~K}$, it was found that well-expressed quantum behavior takes place at temperatures below $13 \mathrm{~K}$, with the lifetime in the excited state exceeding $20 \mathrm{~ms}$ at $5.4 \mathrm{~K}$. This is far superior to modern quantum computers built on conventional Josephson junctions.

The threshold temperature of $13 \mathrm{~K}$ in [6] is $14.4 \%$ of the critical temperature of $\mathrm{YBa}_{2} \mathrm{Cu}_{3} \mathrm{O}_{7-\mathrm{x}}$. For the $2 \Delta / \mathrm{e}$ of $0.6 \mathrm{~V}$, the critical temperature of superconductor in microtubules present in axons in the brain, was estimated in [3] as $2022 \pm$ $157 \mathrm{~K}$. The room temperature of $295 \mathrm{~K}$ is then $14.6 \%$ of the mean value of the estimated critical temperature in microtubules. Following arguments in [6], it means that quantum processing of information using phase slips in the superconductor is possible at room temperature. It is an additional argument to those presented in [3] in favor of superconductivity as basis of quantum processing of information in living organisms.

\section{B. Origin of superconductivity in microtubules}

According to [3], superconductivity takes place in structured water inside microtubules. One should not be too sceptic about superconductivity in this liquid that is seemingly poorly conductive in its three-dimensional form. On one hand, it is well known that metals with the best conductivity, e.g. gold, silver and copper, are not superconducting. On the other hand, poorly conductive oxides like that investigated in [6] are known for breakthrough critical temperatures in a quasi twodimensional form of superconductivity (see, for example, historical reviews in [3] and [11]). One would also not expect superconductivity with record-high critical temperature at high pressure in $\mathrm{H}_{2} \mathrm{~S}$, a gas that is non-conductive at ambient conditions [12]. The key element for superconductivity in $\mathrm{H}_{2} \mathrm{~S}$ is light hydrogen atoms [12], as is the oxygen in hightemperature superconductors. Combining these elements together and presenting them in quasi one-dimensional form, like in the model of Little [2], leads one to structural water, which, in microtubules, is confined to an extremely narrow channel, with an inner diameter of $15 \mathrm{~nm}$. The exact mechanism of superconductivity in microtubules is not known yet, although it is almost certain that it should be based on electron-electron interaction [2] and perhaps reflect ability of hydrogen to be in anion state, as discussed in [13].

In [6], it was found that superconductivity could be strongly influenced by background radiation. In microtubules, the structured water channel is tightly screened by specific tubulin protein complexes. The enormous complexity of microtubules on the nano-scale level is the price nature is paying for quantum processing of information at ambient temperature. It is a product of billions of years of evolution.

In Figs. 1 to 3, voltage jumps reflect appearance of permanent phase-slip lines. Quantum processing of information does not require these permanent lines. Most likely, it operates by short-lived phase-slip centers in individual microtubules (on the scale down to picoseconds), which are not possible to register by this technique and which seem to be outside of instrumental abilities in modern nanoscience. General principles of the processing, however, could be understood in terms of Josephson oscillations, an approach that is widely used in design of modern superconducting quantum computers.

\section{Role of Josephson oscillations}

Josephson oscillations in quantum superconducting circuits require the presence of barriers or weak links with a potential difference across them. In the case of living organisms, these could be membranes with a built-up voltage, and the circuit is a network of quasi onedimensional superconducting filaments (microtubules). If voltage $\mathrm{V}$ on Josephson junction is below $2 \Delta / \mathrm{e}$, it results in oscillations and an electromagnetic wave with frequency $v$ proportional to the voltage according to the equation:

$$
v=2 \mathrm{eV} / \mathrm{h},
$$


where $\mathrm{h}$ is the Plank constant.

Typical voltages in a human body, for example, vary between 20 and $200 \mathrm{mV}$ [14], with the average membrane potential of about $70 \mathrm{mV}$. These voltages correspond to 4.8 , 48.4 and $16.9 \mathrm{THz}$, respectively, which are in the far, long and mid-infrared parts of electromagnetic wave spectrum. These parts of the spectrum are in the so-called $\mathrm{THz}$ gap region, in which it is currently problematic to generate and detect electromagnetic radiation, so that experimentation is difficult. Nevertheless, these parts of spectrum seem to be in intensive use by living organisms for processing and even exchange of information.

In [6], it was shown that absorption of single optical photons can influence the phase-slip process and quantum state of superconducting nanowires. It is an example of nonresonant absorption, since frequency of optical photons is much higher than the characteristic frequencies of Josephson oscillations in living organisms. Resonant absorption, however, is also possible, and it is likely that different organs in the organisms and even different organisms can efficiently communicate on specific $\mathrm{THz}$ frequencies. Finding these frequencies would be an exciting direction for further studies.

\section{CONCLUSIONS}

Experimental evidence of the formation of phase slip lines in brain slices of mammals is given, which provides an additional argument in favor of a quasi one-dimensional room-temperature superconductivity assigned to water filaments confined to the narrow space of microtubules. The estimation of the critical temperature of the superconductor confirms that, consistent with a recently published study, a quantum regime can be achieved at room temperature, allowing quantum processing of information in living organisms. The Josephson nature of the processing suggests existence of characteristic electromagnetic oscillations at $\mathrm{THz}$ frequencies, which could be used not only for processing of information, but also for communication inside the body and between different organisms.

\section{REFERENCES}

[1] J. Schmalian, "Failed theories of superconductivity," Modern Physics Letters B, vol. 24, pp. 2679-2691, October 2010.

[2] W. A. Little, "Possibility of synthesizing an organic superconductor," Phys. Rev., vol. 134, pp. 1416-1424, June 1964.

[3] P. Mikheenko, "Possible superconductivity in the brain," J. Supercond. Novel Magn., vol. 32, pp. 1121-1134, May 2019.

[4] E. H. Halperin and A. A. Wolf, "Speculations of superconductivity in biological and organic systems," In: Advances in cryogenic engineering, vol. 17, K.D. Timmerhaus, Ed. New York: Springer Science + Business Media, 1972, pp 109-115.

[5] S. Hameroff, "Quantum computation in brain microtubules? The Penrose-Hameroff 'Orch OR' model of consciousness," Phil. Trans. R. Soc. A, vol. 356, pp. 1869-1896, August 1998.

[6] M. Lyatti, M.A. Wolf1, I. Gundareva, M. Kruth, S. Ferrari, R.E. Dunin-Borkowski and C. Schuck, "Energy-level quantization and single-photon control of phase slips in $\mathrm{YBa}_{2} \mathrm{Cu}_{3} \mathrm{O}_{7-\mathrm{x}}$ nanowires," Nat. Commun., vol. 11, 763, February 2020.

[7] M. H. Devoret and R. J. Schoelkopf, "Superconducting circuits for quantum information: an outlook," Science, vol. 339, pp. 1169-1174, March 2013.

[8] A.K. Geim, K.S. Novoselov, "The rise of graphene," Nature Materials, vol. 6, pp. 183-191, March 2007.

[9] W. J. Skocpol, M. R. Beasley and M. Tinkham, "Phase-slip centers and nonequilibrium processes in superconducting tin microbridges," J. Low. Temp. Phys., vol 16, pp. 145-167, July 1974.

[10] Y. M. Ivanchenko, P. N. Mikheenko and V. F. Khirnyi, "Kinetics of the destruction of superconductivity by the current in the thin films," Sov. Phys. JETP, vol. 53, pp. 86-91, January 1981.

[11] V. Z. Kresin, "Paths to Room-Temperature Superconductivity," J. Supercond. Nov. Magn., vol 31, pp. 611-617, March 2018.

[12] A. P. Drozdov, M. I. Eremets, I. A. Troyan, V. Ksenofontov and S. I. Shylin, "Conventional superconductivity at 203 kelvin at high pressures in the sulfur hydride system," Nature, vol. 525, pp. 73-76, August 2015.

[13] P. Mikheenko, E. M. Baba and S. Karazhanov, "Electrical and Magnetic Behavior of GdOH Thin Films: A Search for Hydrogen Anion Superconductivity," In Microstructure and Properties of Micro- and Nanoscale Materials, Films, and Coatings (NAP 2019), A. D. Pogrebnjak and O. Bondar Eds. Singapore: Springer Nature, 2020, pp. 1-7.

[14] G. J. Tortora and S. R. Grabowski, Principles of Anatomy and Physiology, 7th ed. New York: HarperCollins, 1993. 